\title{
Special Dio 3 - tuples M.A.Gopalan ${ }^{1 *}$, K.Geetha ${ }^{2}$, Manju Somanath ${ }^{3}$ \\ 1. Professor, Department of Mathematics, Shrimathi Indira Gandhi College, Trichy, 2. Assistant Professor, Department of Mathematics, Cauvery College for Women, Trichy, 3. Assistant Professor, Department of Mathematics, National College, Trichy,
}

Keywords: Dio 3 - tuples, Pell equation, Polygonal numbers and Centered polygonal numbers

\begin{abstract}
We search for three distinct polynomials with integer coefficient such that the product of any two members of the set minus their sum and increased by a non- zero integer (or polynomial with integer coefficient) is a perfect square.
\end{abstract}

\section{Introduction:}

The problem of constructing the set with property that the product of any two its distinct elements is one less than a square has a very long history and such sets were studied by Diophantus. A set of $\mathrm{m}$ positive integers $\left\{a_{1}, a_{2}, \ldots a_{m}\right\}$ is called a Diophantine m-tuple

$$
a_{i} * a_{j}+1 \text { is a perfect square, }
$$

a perfect square for all $1 \leq i \prec j \leq m$. Many generalizations of this problem (1) were considered since antiquity, for example by adding a fixed integer $n$ instead of 1 , looking kth powers instead of squares or considering the powers over domains other than $\mathrm{Z}$ or Q. Many mathematicians consider the problem of the existence of Diophantine quadruples with the property $D(n)$ for any arbitrary integer $\mathrm{n}$ and also for any linear polynomials in $\mathrm{n}$. In this context one may refer [1-16]. The above results motivated us the following definition:

A set of three distinct polynomials with integer coefficient $\left(a_{1}, a_{2}, \mathrm{a}_{3}\right)$ is said to be a special dio 3- tuple with property $D(n)$ if $a_{i} * a_{j}-\left(a_{i}+a_{j}\right)+n$ is a perfect square for all $1 \leq i \prec j \leq 3$.

In the above definition $\mathrm{n}$ may be a non - zero integer or polynomial with integer coefficients. In this communication we consider a few special dio 3 tuples of polygonal numbers from $t_{6, n}$ to $t_{10, n}$ and centered polygonal numbers from $c t_{6, n}$ to $c t_{10, n}$ with their corresponding properties.

\section{Notations:}

$$
\begin{aligned}
& t_{m, n}=n\left(1+\frac{(n-1)(m-2)}{2}\right)= \\
& c t_{m, n}=\frac{m n(n+1)}{2}+1=\text { Polygonal number of rank } \mathrm{n} \text { with sides } \mathrm{m}
\end{aligned}
$$

\section{Construction of Dio 3 - tuples for Hexagonal numbers}

Let $a=t_{6, n}, \quad b=t_{6, n-2}$ be Hexagonal number of rank $\mathrm{n}$ and $\mathrm{n}-2$ respectively such that $a b-(a+b)+4 n^{2}-10 n+11$ is a perfect square say $\gamma^{2}$

Let $\mathrm{c}$ be any non zero integer such that 


$$
\begin{aligned}
& a c-(a+c)+4 n^{2}-10 n+11=\alpha^{2} \\
& \mathrm{~b} c-(\mathrm{b}+c)+4 n^{2}-10 n+11=\beta^{2}
\end{aligned}
$$

On solving equations (2) and (3), we get

$$
(b-1) \alpha^{2}-(a-1) \beta^{2}=(a-b)+\left(4 n^{2}-10 n+11\right)(b-a)
$$

Assuming $\alpha=x+(a-1) T$ and $\beta=x+(b-1) T$, in (4) it reduces to

$$
x^{2}=(b-1)(a-1) T^{2}+\left(4 n^{2}-10 n+10\right)
$$

The initial solution of equation (5) is given by

$$
T_{o}=1 \text { and } x_{0}=n^{2}-5 n+1
$$

Therefore,

$$
\alpha=4 n^{2}-6 n
$$

On substuting the values of $\alpha$ and $a$ in equation (2), we get

\begin{tabular}{|c|c|c|c|}
\hline $\mathbf{a}$ & b & c & $D(n)$ \\
\hline \multirow{2}{*}{$t_{6, n}$} & $t_{6, n-2}$ & $t_{18, n}-13 n+9$ & $D(10)$ \\
\hline & $t_{6, n-1}$ & $t_{6,2 n-2}+6 n-4$ & $D\left(10 n^{2}-15 n+7\right)$ \\
\hline \multirow{3}{*}{$2 t_{7, n}$} & \multirow{2}{*}{$2 t_{7, n-2}$} & $2 t_{7,2 n-2}-6 n+5$ & $D\left(10 n^{2}-26 n+35\right)$ \\
\hline & & $t_{42, n}-33 n+29$ & $D(30)$ \\
\hline & $2 t_{7, n-1}$ & $2 t_{7,2 n-2}+14 n+13$ & $D\left(25 n^{2}-40 n+17\right)$ \\
\hline \multirow{3}{*}{$t_{8, n}$} & \multirow{2}{*}{$t_{8, n-2}$} & $t_{8,2 n-2}-4 n+7$ & $D\left(18 n^{2}-48 n+32\right)$ \\
\hline & & $t_{26, n}-21 n+7$ & $D(17)$ \\
\hline & $t_{8, n-1}$ & $t_{8,2 n-2}+8 n-6$ & $D\left(18 n^{2}-30 n+14\right)$ \\
\hline \multirow{3}{*}{$2 t_{9, n}$} & \multirow{2}{*}{$2 t_{9, n-2}$} & $2 t_{9,2 n-2}-10 n+11$ & $D\left(28 n^{2}-76 n+74\right)$ \\
\hline & & $t_{58, n}-49 n+45$ & $D(54)$ \\
\hline & $2 t_{9, n-1}$ & $2 t_{9,2 n-2}+18 n-19$ & $D\left(35 n^{2}-60 n+28\right)$ \\
\hline \multirow{3}{*}{$t_{10, n}$} & \multirow{2}{*}{$t_{10, n-2}$} & $t_{10,2 n-2}+6 n-1$ & $D\left(24 n^{2}-66 n+47\right)$ \\
\hline & & $t_{34, n}-29 n+25$ & $D(26)$ \\
\hline & $t_{10, n-1}$ & $t_{10,2 n-2}+10 n-10$ & $D\left(20 n^{2}-35 n+16\right)$ \\
\hline
\end{tabular}

$$
\begin{aligned}
c & =8 n^{2}-20 n+11 \\
& =t_{6,2 n-2}-2 n+1
\end{aligned}
$$

Therefore, triple $\left(t_{6, n}, t_{6, n-2}, t_{6,2 n-2}-2 n+1\right)$ is Dio 3- tuple with property $D\left(4 n^{2}-10 n+11\right)$

For simplicity, we present in the table below a few Dio 3-tuples for polygonal numbers from $t_{6, n}$ to $t_{10, n}$ with suitable properties. 


\section{Construction of Dio 3 - tuples for Centered Hexagonal numbers}

Let $a=c t_{6, n}, \quad b=c t_{6, n-2}$ be Centered Hexagonal number of rank $\mathrm{n}$ and $\mathrm{n}-2$ respectively such that $a b-(a+b)+24 n^{2}-30 n+2$ is a perfect square say $\gamma^{2}$

Let $\mathrm{c}$ be any non zero integer such that

$$
\begin{aligned}
& a c-(a+c)+24 n^{2}-30 n+2=\alpha^{2} \\
& \mathrm{~b} c-(\mathrm{b}+c)+24 n^{2}-30 n+4=\beta^{2}
\end{aligned}
$$

On solving equations (8) and (9), we get

$$
(b-1) \alpha^{2}-(a-1) \beta^{2}=(a-b)+\left(24 n^{2}-30 n+2\right)(b-a)
$$

Assuming $\alpha=x+(a-1) T$ and $\beta=x+(b-1) T$ in (10), it reduces to

$$
x^{2}=(b-1)(a-1) T^{2}+\left(24 n^{2}-30 n+1\right)
$$

The initial solution of equation (11) is given by

$$
T_{o}=1 \text { and } x_{0}=3 n^{2}-3 n+1
$$

Therefore, $\quad \alpha=6 n^{2}+1$

On substuting the values of $\alpha$ and $a$ in equation (8), we get

$$
\begin{aligned}
c & =12 n^{2}-12 n+9 \\
& =c t_{6,2 n-2}+6 n+8
\end{aligned}
$$

\begin{tabular}{|c|c|c|c|}
\hline a & b & $\mathrm{C}$ & D(n) \\
\hline \multirow[b]{2}{*}{$c t_{6, n}$} & $c t_{6, n-2}$ & $c t_{24, n}-24 n$ & $D(10)$ \\
\hline & $c t_{6, n-1}$ & $c t_{6,2 n-2}+14 n-4$ & $D\left(-12 n^{3}+19 n^{2}-4 n+2\right)$ \\
\hline \multirow{3}{*}{$2 c t_{7, n}$} & \multirow{2}{*}{$2 c t_{7, n-2}$} & $2 c t_{7,2 n-2}+14 n+9$ & $D\left(140 n^{2}-140 n+2\right)$ \\
\hline & & $c_{56, n}-56 n+4$ & $D(22)$ \\
\hline & $2 c t_{7, n-1}$ & $2 c t_{7,2 n-2}+32 n-9$ & $D\left(-70 n^{3}+88 n^{2}-20 n+4\right)$ \\
\hline \multirow{3}{*}{$c t_{8, n}$} & \multirow{2}{*}{$c t_{8, n-2}$} & $c t_{8,2 n-2}+8 n-8$ & $D\left(40 n^{2}-40 n+2\right)$ \\
\hline & & $c t_{32, n}+32 n$ & $D(17)$ \\
\hline & $c t_{8, n-1}$ & $c t_{8,2 n-2}+20 n-4$ & $D\left(-16 n^{3}+28 n^{2}-4 n+2\right)$ \\
\hline \multirow{3}{*}{$2 c t_{9, n}$} & \multirow{2}{*}{$2 c t_{9, n-2}$} & $2 c t_{9,2 n-2}+18 n+11$ & $D\left(234 n^{2}-234 n+7\right)$ \\
\hline & & $c t_{72, n}-72 n+4$ & $D(46)$ \\
\hline & $2 c t_{9, n-1}$ & $2 c t_{9,2 n-2}+24 n-13$ & $D\left(-126 n^{3}+148 n^{2}-28 n+4\right)$ \\
\hline \multirow{3}{*}{$c t_{10, n}$} & \multirow{2}{*}{$c t_{10, n-2}$} & $c t_{10,2 n-2}+10 n+2$ & $D\left(60 n^{2}-60 n+2\right)$ \\
\hline & & $c t_{40, n}-40 n$ & $D(26)$ \\
\hline & $c t_{10, n-1}$ & $c t_{10,2 n-2}+26 n-8$ & $D\left(-20 n^{3}+39 n^{2}-4 n+2\right)$ \\
\hline
\end{tabular}

Therefore, triple $\left(c t_{6, n}, \mathrm{c} t_{6, n-2}, \mathrm{c} t_{6,2 n-2}+6 n+8\right)$ is Dio 3- tuple with property $D\left(24 n^{2}-30 n+2\right)$

For simplicity, we present in the table below a few Dio 3- tuples for Centered polygonal numbers from $c t_{6, n}$ to $c t_{10, n}$ with suitable properties. 


\section{Conclusion:}

In this paper we have presented a few examples of constructing a special Dio 3 tuples for polygonal numbers and centered polygonal numbers with suitable properties. To conclude one may search for Dio 3 - tuples for higher order polygonal numbers and centered polygonal numbers with their corresponding suitable properties.

1* The financial support from the UGC, New Delhi (F.MRP - 5122/14 (SERO/UGC) dated March 2014) for a part of this work is gratefully acknowledged.

\section{References:}

[1]. A.F.Beardon and M.N.Deshpande (2007), Diophantine triples, The mathematical Gazette, 86 (2002), 258- 260.

[2]. Bugeaud, A.Dujella and M.Mignotte (2007), On the family of Diophantine triples $\left\{k-1, k+1,16 k^{3}-4 k\right\}$, Glasgow Math.J., 49, 333-344.

[3]. Lj.Bacic, A.Filipin (2013), On the family of D(4)- triples $\left\{k-2, k+2,4 k^{3}-4 k\right\}$, Bull. Belg. MTH. Soc. Simon Stevin 20, 777- 787.

[4]. Lj.Bacic, A.Filipin (2013), On the extendibility of D(4)- pairs, Math. Commun. 18, 447 -456.

[5]. M.N.Deshpande (2002), One interesting family of Diophantine triplets, International J.Math.ed. Sci.Tech., 33, 253-256.

[6]. M.N.Deshpande (2003), Families of Diophantine triplets, Bulletin of the Marathwada Mathematical Society, 4, 19-21.

[7]. Y.Fujita (2008), The extensibility of Diophantine pairs $\{k-1, k+1\}$, J.Number theory, 128, 322-353.

[8]. Y.Fujita, A.Togbe (2011), Uniqueness of the extension of the $D\left(4 k^{2}\right)-$ triple $\left\{k^{2}-4, k^{2}, 4 k^{2}-4\right\}$, Notes Number Theory Discrete Math.17, 42-49.

[9]. M.A.Gopalan and V.Pandichelvi (June 2009), On the extensibility of the Diophantine triple involving Jacobsthal numbers $\left(j_{2 n-1}, j_{2 n+1}-3,2 j_{2 n}+j_{2 n-1}+j_{2 n+1}-3\right)$, International journal of Mathematics \& Application, 2 (1), 1-3.

[10]. M.A.Gopalan and G.Srividhya (2009), Diophantine Qudrapules for Fibonacci numbers with property D(1), Indian Journal of Mathematics and mathematical Sciences, 5 (2), 57-59.

[11]. M.A.Gopalan and G.Srividhya (2010), Diophantine Qudrapules for Pell numbers with property D(1), Antarctica Journal of Mathematics, 7(3), 357-362.

[12]. M.A.Gopalan and V.Pandichelvi (2011), Construction of the Diophantine triple involving Polygonal numbers, Impact J.Sci.Tech., 5(1), 7-11.

[13]. M.A.Gopalan and G.Srividhya (2012), Two special Diophantine Triples, Diophntus J.Math.,1(1), 23-37.

[14]. M.A.Gopalan, V.Sangeetha and Manju Somanath (2014), Construction of the Diophantine polygonal numbers, Sch. J.Eng. Tech. 2, 19 -22.

[15]. M.A.Gopalan, K.Geetha and Manju Somanath (2014), On special Diophantine Triple, Archimedes Journal of Mathematics, 4(1), 37-43.

[16]. L.Szalay, V.Ziegler (2013), On an S-unit variant of Diophantine m-tuples, Publ. Math. Debrecen 83, 97 -121. 